\title{
Utilising a Loop Structure to Allow a Microfiber Coupler with Larger Taper Diameters to be Used for Sensing
}

\author{
Fangfang Wei \\ Technological University Dublin, fangfang.wei@tudublin.ie \\ Gerald Farrell \\ Technological University Dublin, gerald.farrell@tudublin.ie \\ Qiang Wu \\ Northumbria University, qiang.wu@tudublin.ie
}

See next page for additional authors

Follow this and additional works at: https://arrow.tudublin.ie/engscheleart2

Part of the Electrical and Computer Engineering Commons

\section{Recommended Citation}

Wei, F. (2016) Utilising a loop structure to allow a microfiber coupler with larger taper diameters to be used for sensing, 6th European Workshop on Optical Fibre Sensors (EWOFS), vol. 9916, 991608, Limerick, Ireland (2016) doi: 10.1117/12.2236636

This Article is brought to you for free and open access by the School of Electrical and Electronic Engineering at ARROW@TU Dublin. It has been accepted for inclusion in Articles by an authorized administrator of ARROW@TU Dublin. For more information, please contact arrow.admin@tudublin.ie, aisling.coyne@tudublin.ie, gerard.connolly@tudublin.ie.

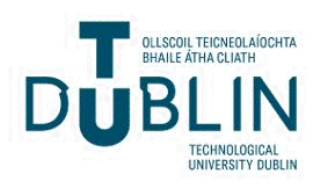




\section{Authors}

Fangfang Wei, Gerald Farrell, Qiang Wu, and Yuliya Semenova

This article is available at ARROW@TU Dublin: https://arrow.tudublin.ie/engscheleart2/222 


\title{
Utilising a loop structure to allow a microfiber coupler with larger taper diameters to be used for sensing
}

\author{
Fangfang Wei ${ }^{1 \mathrm{a}}$, Gerald Farrell ${ }^{\mathrm{a}}$, Qiang $\mathrm{Wu}^{\mathrm{b}}$, Yuliya Semenova ${ }^{\mathrm{a}}$ \\ ${ }^{a}$ Photonics Research Centre, School of Electrical and Electronic Engineering, Dublin Institute of \\ Technology, Kevin St, Dublin 8, Ireland \\ ${ }^{\mathrm{b}}$ Department of Physics and Electrical Engineering, Northumbria University, Newcastle Upon Tyne, \\ NE1 8ST, United Kingdom
}

\begin{abstract}
This paper examines a technique that utilizes a Sagnac loop with a microfiber coupler (MFC) as a coupler which allows the MFC to operate effectively as a sensor but with larger than normal tapered fiber diameters. The proposed structure is found to be suitable for temperature and refractive index (RI) sensing. It is shown that a variation in the surrounding of the MFC RI results in a shift of the output spectrum, while a temperature variation leads to changes in the intensity of the interference dips. A decrease in the waist diameter of the MFC results in an increase in the sensitivity to temperature. For MFC structures based on a $5.6 \mu \mathrm{m}$ and a $3 \mu \mathrm{m}$ fiber waist diameter, the minimum transmission power level of a selected spectral dip decreases by $1.7 \mathrm{~dB}$ and $5.03 \mathrm{~dB}$ respectively, as the temperature changes from $18{ }^{\circ} \mathrm{C}$ to $44{ }^{\circ} \mathrm{C}$. A change in the surrounding RI from 1.334 to 1.395 results in the spectral redshift of $8 \mathrm{~nm}$ using a $5.6 \mu \mathrm{m}$ fiber waist diameter. By functionalizing the surface of the MFC with various materials, the structure could potentially be used for sensing of other parameters.
\end{abstract}

Keywords: Microfiber coupler (MFC), fiber loop interferometer, temperature sensor, RI sensor, microfiber sensor

\section{INTRODUCTION}

The fiber loop mirror, or a Sagnac interferometer, has aroused significant research interest over many years, due to its stability, flexibility, and ease of fabrication ${ }^{1}$. The operating principle of the Sagnac loop is based on the multi-beam interference between clockwise and counterclockwise beams in the fiber loop ${ }^{2}$. Sagnac loops can be used as filters in various devices for optical communications ${ }^{3}$ and as fiber optic sensors for strain, temperature and other physical parameters ${ }^{4}$. Optical microfiber couplers (MFCs) have also become a topic of special interest in optical fiber sensing due to their superior sensing properties compared with conventional fused fiber couplers ${ }^{5}$. The value of the tapered fibre diameters and the degree of fusion determines whether the fibers forming the MFC are strongly or weakly fused. The former case is more suited to work as a beam combiner and splitter for optical fiber communications, and the latter shows a strong potential for sensing applications ${ }^{6}$.

The mechanical stability of MFCs is a challenge, which becomes more problematic as the waist diameter of the tapered section of the fibers decreases. Typically for effective sensing, tapered fiber diameters used in MFCs need to reach values close to or below $1 \mu \mathrm{m}$, which are mechanically less stable. This paper examines a technique that utilizes a Sagnac loop with an MFC as a coupler, that allows the MFC to operate effectively as a sensor but with tapered fiber diameters circa $5 \mu \mathrm{m}$.

The structure proposed here combines a Sagnac loop and an MFC. In this structure an MFC is substituted for a traditional $3 \mathrm{~dB}$ coupler in the fiber loop mirror to form a Sagnac interferometer in which the MFC serves as a sensing head. When a broadband light is launched into such a fiber loop, any change in ambient conditions such as temperature, polarization, strain and refractive index (RI) affecting the MFC will result in changes in the output interference spectrum. Reducing the diameter of the MFC leads to an increase of the evanescent field and this supports stronger lightenvironment interactions as well as the basic coupler function.

In this paper the aim is to investigate the properties of a Sagnac interferometer based on a fiber loop mirror combined with a tapered MFC serving both as a beam combiner and a sensing head, with the objective of allowing larger fiber taper waist diameters to be used. The waist diameter of the MFC is crucial to the operation of the structure. If the MFC

\footnotetext{
${ }^{1}$ fangfang.wei@mydit.ie; phone +353 14024812; fax +353 1402 4690; www.prc.dit.ie
} 
waist diameter is greater than $10 \mu \mathrm{m}$, it operates only as the beam combiner and shows little responsiveness to surrounding changes. If the MFC waist diameter is less than $3 \mu \mathrm{m}$, the overall optical loss is rather high and the interference spectrum is unstable. In addition, MFCs based on fibers with such small waist diameters are very fragile. Observation of the spectrum using an optical spectrum analyzer (OSA) during the fabrication of the MFC is thus used in our experiment to control the tapering process by observing the spectrum in realtime. Here we present the results of experimental investigations of the influence of temperature and the surrounding RI on the properties of the proposed structure to determine if the structure can operate as an effective sensor and as a precursor to future sensing applications, for example by functionalizing the surface of the MFC with various materials, the structure could potentially be used for sensing of humidity, electric and magnetic fields and chemical quantities.

\section{OPERATING PRINCIPLE AND MFC FABRICATION}

A schematic diagram of the proposed structure and its characterization setup are shown in Fig. 1. The fiber loop mirror interferometer consists of a tapered MFC, a $20 \mathrm{~cm}$ long section of polarization maintaining fiber (PMF) forming a loop and a fiber based polarization controller (PC). Once the fabrication of the MFC is finished, the PC is set to a certain point so as to make sure there is no polarization state change in the system. The MFC is fabricated by simultaneously tapering and fusing two standard single-mode fibers (SMF-28) using a method known as the microheater brushing technique ${ }^{7}$. A broadband source (BBS) with a bandwidth from $1030 \mathrm{~nm}$ to $1660 \mathrm{~nm}$ serves as the light source and the output spectrum is observed using an optical spectrum analyzer (OSA) with a resolution of $20 \mathrm{pm}$. An isolator (ISO) is inserted between the BBS and MFC for protection purposes to prevent backward light reaching the BBS.

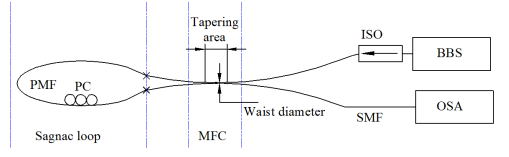

Fig. 1. Schematic diagram of the proposed structure and its characterization setup.

One function of the MFC is to split the light into two light signals which counter propagate in the PMF loop. But unlike a conventional coupler used in such a structure, when input light enters the MFC, since the evanescent field extends into the surrounding medium, changes in the surrounding medium will affect the coupling coefficient of the MFC and thus the phase of the light entering the two ports of the PMF loop. Within the PMF loop the two light signals counter propagate, accumulating a phase difference in the PMF before combining again in the MFC. Within the MFC, the returning light signals recombine and interfere because of the fixed phase difference acquired over the PMF loop. As the returning signals recombine and interfere in the MFC, then once again the surrounding environment influences this process, for example by influencing the coupling ratio. To illustrate this further the coupling coefficient of the MFC in this structure can be approximately denoted as .

$$
R(\lambda)=\frac{\pi \sqrt{n_{1}^{2}-\mathrm{n}_{2}^{2}}}{2 a n_{1}} \mathrm{e}^{-2.3026\left(\mathrm{~A}+\mathrm{B} \tau+\mathrm{C} \tau^{2}\right)}
$$

where $\lambda$ is the wavelength, $\mathrm{n}_{1}$ and $\mathrm{n}_{2}$ are the RIs of the fiber and surrounding medium, $a$ is the radius of the microfiber, $\tau$ is the aspect ratio of the cross section of MFC and A, B, C is the non-homogeneous term of the coefficient matrix for the normalized frequency equations ${ }^{7}$. From equation (1), it can be seen that the MFC acts as a sensing head, since surrounding environmental parameters such as temperature and RI will have influence on the coupling ratio of the MFC and the phase.

In these experiments, the PMF loop is setup so as to avoid temperature effects by ensuring the PMF is isolated from temperature variations, and polarization is controlled by setting the PC to a defined state; thus only the MFC works as the sensing component.

Initially four separate MFC samples with minimum tapered fiber waist diameters of $10 \mu \mathrm{m}, 7 \mu \mathrm{m}, 5.6 \mu \mathrm{m}$ and $3 \mu \mathrm{m}$ were fabricated and characterized using the setup in Fig. 1. It should be noted that for each of the MFC samples the output spectrum was constantly monitored throughout the tapering process to ensure efficient fusing of the fibers and quality of the resultant interference spectrum. Fig. 2 illustrates an example of experimentally measured transmission spectrum for the fiber loop mirror with a $5.6 \mu \mathrm{m}$ waist diameter MFC. Interference dips are clearly observed and evenly spaced. This is expected as the output spectrum of a traditional Sagnac loop structure is approximately a periodic function of the inverse wavelength. The free spectral range (FSR) of the spectrum is estimated as $14 \mathrm{~nm}$. In practice, while four samples were fabricated, only the samples with fiber waist diameters of $5.6 \mu \mathrm{m}$ and $3 \mu \mathrm{m}$ were used in further experiments, as these MFCs demonstrated spectra that were most sensitive to the local environment. 


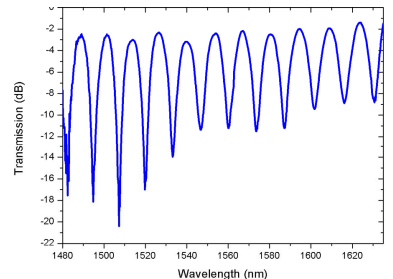

Fig. 2. Experimental spectrum of the microfiber loop sensor with a $5.6 \mu \mathrm{m}$ fiber waist diameter MFC.

\section{EXPERIMENTS}

Studies of the influence of temperature and surrounding refractive index on the output transmission spectrum of the proposed fiber loop mirror structure were carried out using the basic setup in Fig. 1 for the two sample structures containing MFCs with different fiber waist diameters.

\subsection{Temperature response}

To measure the temperature response, a heater is placed close to the tapered point of the MFC while keeping the other parts of the structure a distance apart and thus at room temperature $\left(18^{\circ} \mathrm{C}\right)$. The temperature is increased from $18^{\circ} \mathrm{C}$ to $44^{\circ} \mathrm{C}$ in steps of $1{ }^{\circ} \mathrm{C}$. Fig. 3(a) shows a series of experimental spectra recorded at different temperature points for the structure based on the MFC with a fiber waist diameter of $5.6 \mu \mathrm{m}$, and Fig. 3(b) shows the relationship between temperature and transmission power in wavelength of $1573.42 \mathrm{~nm}$.

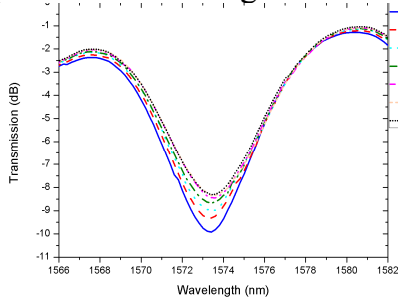

a)
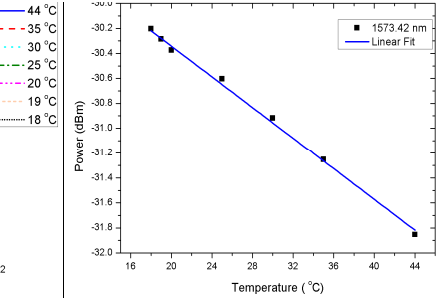

b)

Fig. 3. (a) Transmission spectra for the structure based on a MFC with $5.6 \mu \mathrm{m}$ fiber waist diameter at different temperatures;

(b) spectra power versus temperature.

As one can see from the figure the most notable changes due to temperature occur in the power levels of the interference minima. As the temperature increases from $18{ }^{\circ} \mathrm{C}$ to $44{ }^{\circ} \mathrm{C}$ the dip transmission power decreases by circa $1.7 \mathrm{~dB}$. As expected, a decrease in the waist diameter of the MFC utilized within the proposed fiber configuration results in a greater sensitivity to temperature. This is illustrated in Fig. 4 (a), which shows a similar dependence for the structure containing a $3 \mu \mathrm{m}$ waist diameter MFC. The change in the dip transmission power in the same temperature range is almost $5.03 \mathrm{~dB}$. Optical loss was increased and mode hoping occurred in the interference spectrum with smaller diameter MFC. Fig. 4(b) shows the relationship between temperature and transmission power at a wavelength of $1571.8 \mathrm{~nm}$.

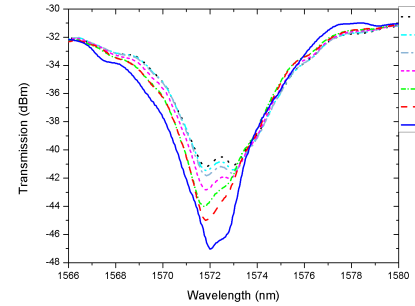

a)

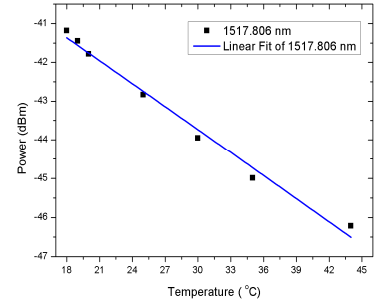

b)

Fig. 4. (a) Transmission of a single spectral dip measured at different temperatures for the structure based on a MFC with 3 $\mu \mathrm{m}$ fiber waist diameter; (b) spectra power versus temperature.

\subsection{Refractive Index response}

In order to measure the refractive index response, a Dimethyl Sulfoxide-water mixed solution with different concentrations was used as the samples for an RI range from 1.334 to 1.395, measured independently using an Abbe refractometer. Fig. 5 shows the results of the RI experiments for the structure based on the $5.6 \mu \mathrm{m}$ fiber waist diameter MFC. The experimental transmission spectra recorded when liquids with different RIs were applied to the MFC waist 
are shown in Fig. 5(a). It is clear that an increase in the surrounding RI leads to a linear redshift of the transmission spectrum. This is summarized in Fig. 5(b), which shows the dependence of spectral dip position versus surrounding RI for the three selected dips.

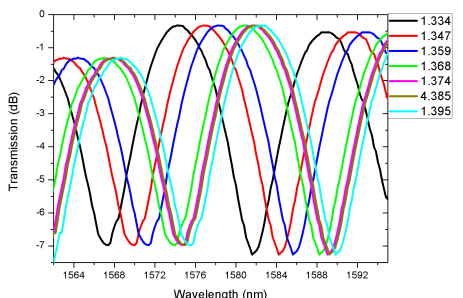

a)

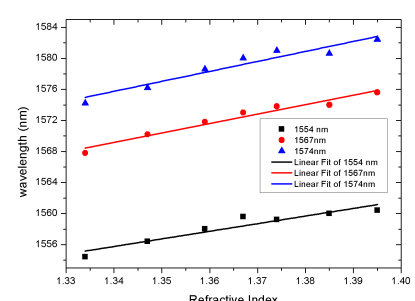

b)

Fig. 5. (a) RI response of the structure with a $5.6 \mu \mathrm{m}$ fiber waist diameter MFC in the range from 1.334 to 1.395 ; (b) spectral dip position versus surrounding RI.

The overall spectral redshift when the surrounding RI changes from 1.334 to 1.395 is estimated as $8 \mathrm{~nm}$ resulting in RI sensitivity of $134.42 \mathrm{~nm} / \mathrm{RIU}$ in the wavelength of $1572 \mathrm{~nm}$.

\section{CONCLUSION}

A tapered microfiber coupler loop structure for sensing has been proposed and studied. The results indicate that the structure is suitable for temperature and RI sensing. It is shown that a variation in the surrounding RI results in a shift of the output spectrum, while temperature variations lead to changes in the minimum transmission of the interference dips. A decrease in the waist diameter of the MFC results in an increase of the sensitivity to temperature. For a structure based on a $3 \mu \mathrm{m}$ waist diameter MFC, the minimum transmission power level of a selected spectral dip decreases by $5.03 \mathrm{~dB}$ as the temperature changes from $18{ }^{\circ} \mathrm{C}$ to $44{ }^{\circ} \mathrm{C}$. For the $5.6 \mu \mathrm{m}$ waist diameter MFC, a change in the surrounding RI from 1.334 to 1.395 results in the spectral redshift of $8 \mathrm{~nm}$, resulting in the corresponding sensitivities of $0.16 \mathrm{~dB} /{ }^{\circ} \mathrm{C}$ and $134.42 \mathrm{~nm} / \mathrm{RIU}$ at a wavelength of $1572 \mathrm{~nm}$. The proposed structure offers the advantages of better mechanical stability compared with "thin" MFCs with waist diameters less than $3 \mu \mathrm{m}$, and the possibility of sensing temperature and RI simultaneously by measurements of the spectral intensity variation and spectral shift. By functionalizing the surface of the MFC with various materials, the structure could potentially be used for sensing of biological, chemical quantities and or electromagnetic fields.

\section{REFERENCES}

1. L. Y. Shao, Y. Luo, Z. Y. Zhang, X. H. Zou, B. Luo, W. Pan, L.S. Yan, "Sensitivity-enhanced temperature sensor with cascaded fiber optic Sagnac interferometers based on Vernier-effect," Opt. Commun. 336, 73-76 (2015).

2. D. P. Zhou, L. Wei, W. K. Liu, J. W.Y. Lit., " Simultaneous measurement of strain and temperature based on a fiber Bragg grating combined with a high-birefringence fiber loop mirror," Opt. Commun. 281(18), 4640-4643 (2008).

3. X.P. Dong, S. Li, K.S. Chiang, M.N. Ng, B.C.B. Chu, "Multiwavelength erbium-doped fibre laser based on a highbirefringence fibre loop mirror," Electron. Lett. 36(19), 1609 (2000).

4. P. Zu, C. C. Chan, G. W. Koh, W. S. Lew, Y. X. Jin, H. F. Liew, W. C. Wong, W. Y. Dong, "Enhancement of the sensitivity of magneto-optical fiber sensor by magnifying the birefringence of magnetic fluid film with Lyot-Sagnac interferometer," Sensors and Actuators B: Chemical, 191, 19-23 (2014).

5. P. Wang, M. Ding, G. Bramilla, Y. Semenova, Q. Wu, and G. Farrell, "High temperature performance of an optical microfibre coupler and its potential use as a sensor," Electron. Lett. 48 (5), 283-284(2012).

6. R. G. Lamont, D. C. Johnson, and K. O. Hill, "Power transfer in fused biconical-taper single-mode fiber couplers: dependence on external refractive index," Appl. Opt., 24 (3), 327 (1985).

7. B. Lin, "Tapered Optical Microfibre Based Structures for Sensing Applications," Doctoral Dissertation. Dublin Institute of Technology, (2015). 